\title{
Person Identification by Analyzing Door Accelerations in Time and Frequency Domain
}

\author{
Hristijan Gjoreski ${ }^{1 凶}$, Rok Piltaver ${ }^{1,2}$, Matjaž Gams ${ }^{1,2}$ \\ ${ }^{1}$ Jožef Stefan Institute, Ljubljana, Slovenia \\ ${ }^{2}$ Jožef Stefan International Postgraduate School, Ljubljana, Slovenia \\ \{hristijan.gjoreski, rok.piltaver, matjaz.gams\}@ijs.si
}

\begin{abstract}
The paper describes an approach for recognizing a person entering a room using only door accelerations. The approach analyzes the acceleration signal in time and frequency domain. For each domain two types of methods were developed: (i) feature-based - use features to describe the acceleration and then uses classification method to identify the person; (ii) signal-based - use the acceleration signal as input and finds the most similar ones in order to identify the person. The four methods were evaluated on a dataset of 1005 entrances recorded by 12 people. The results show that the time-domain methods achieve significantly higher accuracy compared to the frequency-domain methods, with signalbased method achieving $86 \%$ accuracy. Additionally, the four methods were combined and all 15 combinations were examined. The best performing combined method increased the accuracy to $90 \%$. The results confirm that it is possible to identify a person entering a room using the door's acceleration.
\end{abstract}

Keywords. Unobtrusive person identification, door, acceleration, machine learning, dynamic time warping.

\section{Introduction}

Ambient intelligence (AmI) is a scientific field that refers to environments consisting of smart devices (sensors and actuators) that can sense and respond to the presence of people [1,2]. An AmI system should work in a way that supports people 's everyday life activities in an easy, natural way using information and intelligence that is hidden in the data provided by the sensors. In order to provide the appropriate assistance, the system has to recognize (identify) the user, i.e., to be aware of his/hers presence. This makes the process of person identification one of the most essential and basic building blocks and a key prerequisite for numerous tasks in AmI.

Person identification is a process through which a person is recognized using some information about him/her. This information can be biometrics (fingerprint, iris, 
voice, etc.), token-based (RFID), or using PIN codes. A disadvantage of these, existing identification methods (e.g., fingerprint, RFID, PIN), is that the identified person needs to perform an action (enter a password, perform a fingerprint scan) or carry an identification token (key or identification card).

This paper presents a novel, completely unobtrusive system for automatic person identification. Our approach identifies the person entering a room based on the acceleration produced by the door movement. The main research hypotheses investigated in this paper are: whether each user has a unique way of entering, and whether the proposed methods enable accurate identification. In the experiments, the identification is performed using the acceleration signal recorded by an accelerometer fixed on a door. In particular, the acceleration signal is analyzed in time domain and frequency domain. For each domain two types of methods are tested: (i) feature-based - uses features to describe the acceleration and then uses classification method to identify the person; (ii) signal-based - uses the acceleration signal as input and find the most similar ones in order to identify the person. Additionally, we tested the combinations of the methods and exploited the performance of all 15 combinations.

The motivation for the work comes from the commonly observed phenomena that humans can recognize their friends and family members by their voice, silhouette, or even gait and other characteristic behavior. Furthermore, there are several known methods for person identification based on gait [3] and appearance [4] - both with considerable application limitations. Furthermore, body-worn accelerometers have been successfully applied to detect falls $[5,6]$, recognize person activity $[7,8]$, and estimate energy expenditure $[9,10]$, while accelerometers attached to door are used to recognize door malfunctions [11].

The paper is organized as follows. In the next section, the background of the study is presented: first by giving a brief description of the physics background and description of the acceleration signal, and then by presenting the relevant related work. In the next two sections, 3 and 4, the methods for each of the domains are presented: feature-based and signal-based methods for the time and the frequency domain, respectively. Section 5 describes the experiments, first by describing the experimental dataset and then by giving the experimental results for each of the methods and their combination. Finally, the paper ends with a conclusion and directions for future work.

\section{Background}

\subsection{Physics and Acceleration Signal Description}

Several phases of door movement can be observed as the person enters (as shown in Figure 1). The door is motionless before the entry, therefore minor deviations from the static values of accelerations in each direction are caused only by sensor noise and vibrations due to outside influences. When entering, the person first accelerates the door in order to open it, which is observed as an increase in angle of the door, angular velocity and acceleration. Then, the acceleration starts to decrease and becomes negative until the door stops at the maximum angle. The door can remain in this state for a 
period of time or just an instance. Then the person accelerates the door in the opposite direction (closing), angular velocity becomes negative and the angle starts to decrease. Before the door angle returns to zero (door closed) the person decreases the angular velocity. If the door is not slowed down enough (door is slammed), a period of damped oscillation lasting up to about a second is observed after closing the door. Examples are shown in Figure 1.

The described physical phenomena can be measured using various sensors such as accelerometer, gyroscope, magnetometer, and door-opening angle sensor. The accelerometer was chosen for the experiment because of availability, installation simplicity, past experiences, successful applications, and extensive related work. A 3-axis accelerometer was attached to the door so that one of its axis was aligned with the direction of gravitation, resulting in measurement of gravitation acceleration $a_{g}$. The second axis was parallel and the third perpendicular to the door-plane, resulting in measurements of the radial $a_{r}$ and tangential accelerations $a_{t}$ respectfully. The gravitation acceleration depends only on the geographical position of the door and remains constant during the entry unless the axis become misaligned with direction of gravity, e.g., when the door is slammed hard and starts oscillating. Radial acceleration $a_{r}=$ $\omega^{2} R$ depends on the angular velocity $\omega$ and the distance of the accelerometer from the axis of door rotation $R$. Tangential acceleration $a_{t}=\alpha R$ depends on the angular acceleration $\alpha$ and $R$ (Figure 2).

\section{$2.2 \quad$ Related Work}

There are various approaches to identify a person entering a room, home, or building. The person is usually recognized or authenticated using a sensor placed near the entrance, which may be: a fingerprint scanner, a camera-based face recognition, RFID reader, a PIN pad, or similar. The following paragraphs describe these approaches and explain how they compare to ours.

Fingerprint scanners are one of the most commonly used and well-established identification sensors [12]. With the recent developments in sensor technology, fingerprint scanners are also being used in the high-end smartphones for various security related functions, such as unlocking the phone and authenticating financial transactions [13]. The scanner analyses the person's fingerprint, i.e. the pattern of the ridges and furrows on the surface of the fingerprint, to identify the person. The reason for the success of fingerprint scanners is that each individual has a unique fingerprint, which enables high identification accuracy. Similarly, hand scanners identify the person using a print of the whole hand instead of a single finger. However, there are several limitations for successful identification with a fingerprint or hand scanner: a complete scan with a reliable quality is needed; moisture, sweat and partial scans significantly impair the identification accuracy; and finally, it is obtrusive for the identified person because one has to make an action in order to be identified: take of a glove, wipe out the finger/hand, put the hand on the scanner.

The recent advancements in machine vision enable camera-based approaches for identification of people [14]. This approach analyzes an image of a person's face in order to perform face recognition by matching it to known face images saved in a 
database. Even though this approach can perform without bothering the person, the cameras are not widely accepted due to the intrusion of the person's privacy. Similarly as fingerprint scanners, camera-based face recognition performs poorly with partial face images, and strongly depends on the environment and the light conditions.

Token-based identification is also commonly used. It became attractive with the development of the RFID and Near-Field Communication (NFC) technology [15]. Typically, each user is issued the respective token — for example, an RFID card that contains data indicating the user's identity. This approach is practical for multisite large-scale installations of access-control systems having many access points and users. Even though token-based identification is quite effective and commonly used in practice, it is obtrusive because it requires additional effort from the user upon each entry. Furthermore, it is not as secure as the approaches discussed above, because the user may lose the identification token and any other person having the token can access the facility. A solution to this problem is the use of PIN instead of a card. However, this approach is still considered obtrusive. First, the person has to remember the PIN, which is problematic because users have to remember multiple PINs (credit cards, web-passwords). Second, it has the same drawback as the fingerprint scanner: it requires an additional effort from the user to enter the PIN. Third, it can be stolen.

The main advantage of our approach is that we use a simple, small and inexpensive accelerometer sensor attached to the door. Therefore, the sensing is completely unobtrusive, except the fact that the system needs certain number of recording to be recorded by the people to be recognized before it is applied in practice. To the best of our knowledge, our work is the first attempt to identify a person using door accelerations.

\section{Time Domain Identification}

This section presents the methods that are analyzing the door acceleration data in time domain, i.e., as provided by the accelerometer. An example of the acceleration signal in time domain is given in Figure 1. Two classification methods were implemented:

- Feature-based method. The method first extracts dozens of domain-specific features from the signal, combines them in a feature vector, which is passed to a pretrained classification model to recognize the person.

- Signal-based method. The method takes the signal as input, compares it to the already known signals (training data) and finds the most similar ones. The signal is classified according to the class values of the most similar signals.

\subsection{Feature-based Identification}

The first method, TD-Feature, analyzes the acceleration signal in time domain using domain specific features. That is, the method first uses the raw acceleration data to extract various domain-specific features, then the most relevant features are selected, and finally the person is identified by a previously trained ML classification model.

The identification starts with the feature extraction from the acceleration signal. The method analyzes the acceleration signal for each opening and closing of the door. 
In order to calculate the features, three parameters are considered: radial acceleration $\alpha$, angular velocity $\omega$, and the angle by which the door is opened $\varphi$. These parameters are analyzed in 14 phases as illustrated in Figure 1:

- 1a-b: the start and end of entrance

- 2a-d: door opens, door stops, door closes

- 3a-f: acceleration changes between negative, zero and positive

- 4a-e: acceleration maximums and minimums in each level-3 subsection

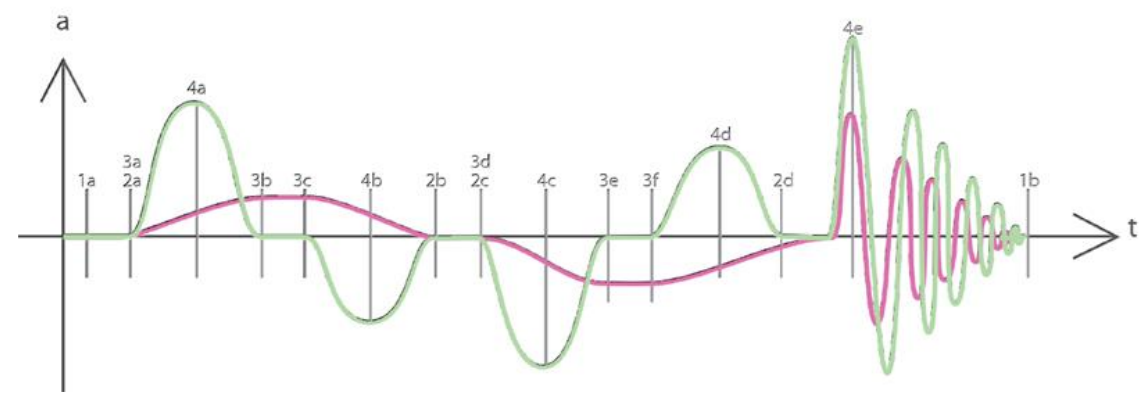

Figure 1 . The 14 phases of the acceleration signal. The $x$ axis shows the time $(t)$ and the $y$ axis shows the acceleration $\alpha$ (green) and the velocity $\omega$ (red).

Besides the duration of each phase, the following features are extracted for each phase and for each parameter $(\alpha, \omega, \varphi)$, resulting in the total of 266 features:

- extreme value of the parameter in the particular phase;

- linear interpolation of the slope of the parameter in the particular phase;

- standard deviation of the parameter in the particular phase; and

- area under the curve (integral) of the parameter in the particular phase.

Because door moves with a single degree of freedom, some of these features are redundant (highly correlated) and some are irrelevant. In order to find and remove the irrelevant and the most correlated (redundant) features, a correlation matrix for all of the features was first calculated and then the most correlated features were removed [16]. This procedure reduced the number of feature from 266 to 97 . After removing the most correlated features, the WrapperSubsetEval (WSE) algorithm [17] was applied, which further reduced the number of features to 36 .

Once the features were extracted and selected, the feature vector was fed into a classification model. The model is trained to identify the person that enters the room according to the feature vector extracted from the acceleration signal. The person's name is used as the class label (the value to be predicted). Four classification algorithms were considered when constructing the model: Decision Tree j48 [18], Random Forest [19], $k$-NN [20], and Support Vector Machine (SVM) [21]. We used the implementation provided in WEKA-ML tool [23]. The results of each algorithm are given in Section 5.1. 


\subsection{Signal-based Identification}

The second method, i.e., TD-Signal, used to identify people entering through the door is based on comparison of the signals (accelerations) in time domain. The method applies dynamic time warping (DTW) to the time-series to calculate their similarity and then uses $k$-nearest neighbors method to identify the person.

Dynamic time warping [22] was successfully applied in many areas such as speech recognition [24], handwriting recognition [25], gesture recognition [26], time-series clustering [27], etc. The DTW algorithm applies non-linear stretching (in the time dimension) to the compared time-series in order to find the optimal match. Figure 2 shows two original time-series (top) and the optimal match between them (bottom) computed using DTW. The optimally time-stretched time series are then used to calculate the dissimilarity of the two time series, i.e. DTW distance.
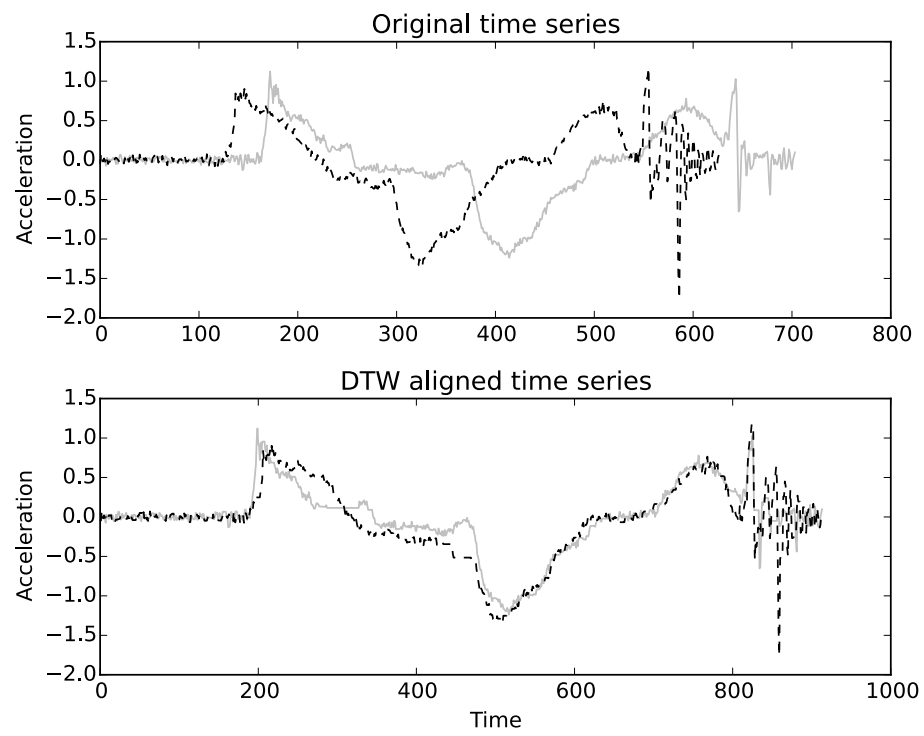

Figure 2. An example of recorded and DTW aligned acceleration during two entries.

A naïve DTW algorithm has $\mathrm{O}(\mathrm{nm})$ time and space complexity where $n$ and $m$ are the lengths of the two compared time series. However, more efficient algorithms such as FastDTW [28] and SparseDTW [29] exist.

The DTW distances between the door-acceleration time-series recorded during the entry and the labeled acceleration time-series of previous entries (labels correspond to persons) are used to identify the person that entered the door using the $k$-nearest neighbors method. Therefore time complexity of this method is $\mathrm{O}(N)$ where $N$ is the number of labeled time-series (i.e. the size of the training set). The time complexity can be reduced by decreasing the number of labeled instances: instead of all the labeled instances only a few entries representing each user are kept.

The labels of the most similar previous entries are finally used to identify the person who entered the door. Two variants of the $k$-nearest neighbors method were compared. The first is to identify the person entering the door as the most frequent label 
among the $k$ most similar previous entries - majority voting. The second variant is to use weighted voting, which considers the more similar entries as more relevant. It does so by assigning them higher voting weight compared to the less similar entries. The weight of the vote $w=1 / d$ for each of the $k$ most similar entries is inversely proportional to its DTW distance $d$ from the identified time series. The label with the highest sum of the votes is the predicted identity of the person who entered. If several labels have an equal sum of votes (in case of weighted voting) or frequency (in case of majority voting), a random label among them is returned as the predicted identity.

\section{Frequency Domain Identification}

This section presents the person identification process that is based on the analysis of the door acceleration data in frequency domain, i.e., the time-domain signal is transformed in frequency domain using Fast Fourier Transform [30]. Figure 3 shows an example of door acceleration during an entrance in time (top-left) and frequency domains (bottom-left).
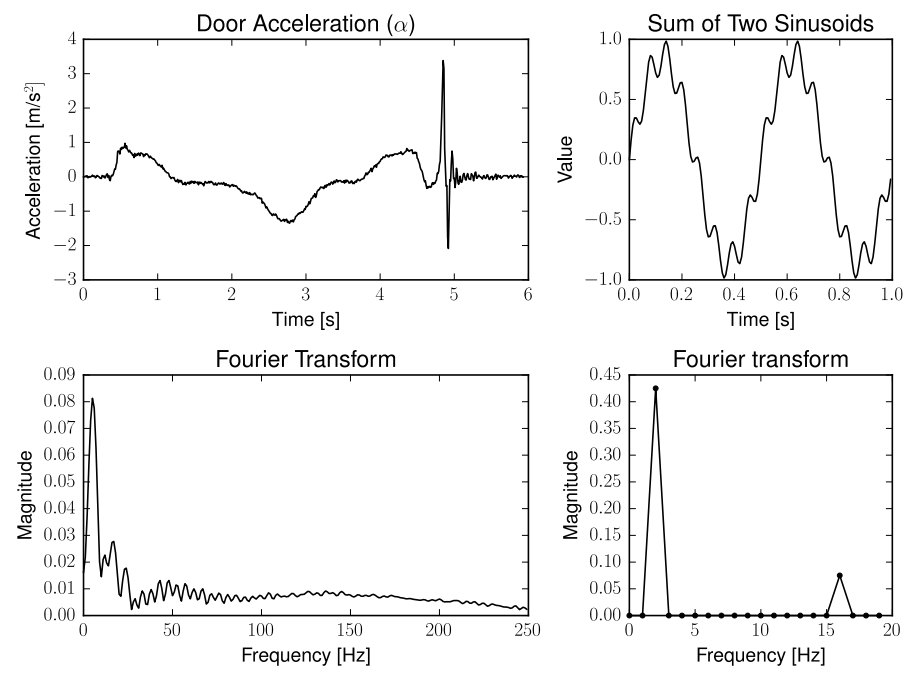

Figure 3. Door acceleration signal (left) and sum of two sinusoids (right) in time (top) and frequency domain (bottom).

The Fourier transform decomposes a function - in our case the sampled angular acceleration of the door - into the composing frequencies. For example, the transform of the signal shown in the top-right graph in Figure 3, given by equation $f(t)=$ $0.85 \sin (t \alpha)+0.15 \sin (8 t \alpha)$, is shown in bottom-right graph in Figure 3 . It clearly shows the two frequencies $(2$ and $8 \times 2 \mathrm{~Hz})$ and their relative amplitudes. Since the acceleration signal is discrete in both time and value, discrete Fourier transform was applied. Similar to the time-domain methods, two methods were developed: featurebased and signal-based methods. 


\subsection{Feature-based Identification}

This section presents the feature-based method that uses the acceleration signal in frequency domain, i.e., FD-Feature method. In order find and calculate the most relevant features, we first computed the average Fourier transform - an example for four persons is shown in Figure 4. The visualization shows that the maximum average magnitude is between 6 and $8 \mathrm{~Hz}$; that a local minimum is between 12 and $18 \mathrm{~Hz}$, and the local maximum is between 19 and $22 \mathrm{~Hz}$. Magnitudes of higher frequencies are considerably lower and local extremes are less distinct. Analysis of the graphs resulted in the definition of the initial set of features: magnitudes of several frequencies below $30 \mathrm{~Hz}$, sums of magnitudes for windows of 10 sequential frequencies below $100 \mathrm{~Hz}$, and sums of magnitudes for larger frequency windows of up to $800 \mathrm{~Hz}$.

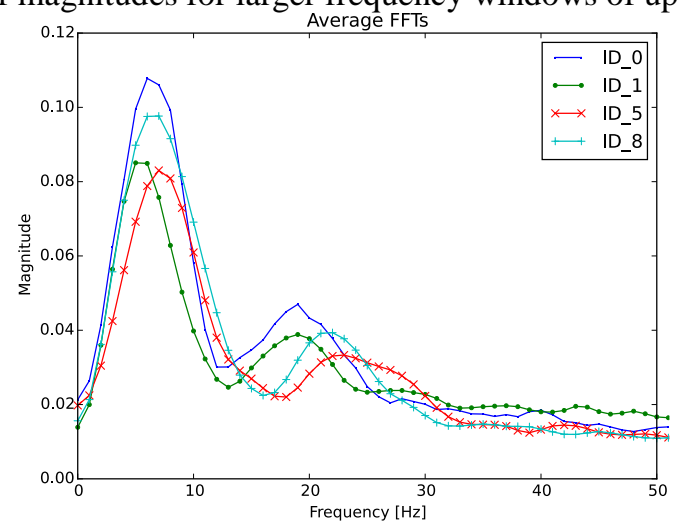

Figure 4: Average Fourier transform of door acceleration for four persons.

The initial set of features was further analyzed using attribute ranking measures, such as ReliefF, Information Gain and Gini Ratio. Additionally, the features were analyzed using the Orange toolkit [31]. For pairs with considerable negative interaction, the feature with the lower feature quality rank was removed from the feature set. For positive interaction and for features with high quality rank, a similar feature was added to the set of features. The interactive process for selecting the feature set was stopped when the accuracy of classification models stopped increasing.

Once the final feature vector was defined, the traditional ML classification process was applied. That is, the selected frequency domain features were extracted and composed into a feature vector, which was fed into a training algorithm that produced a classification model. Similarly to the TD-Feature method, the same four classification algorithms were used: Decision Tree j48 [18], Random Forest [19], $k$-NN [20], and Support Vector Machine (SVM) [21].

\subsection{Signal-based Identification}

The second method developed for the frequency domain, FD-Signal method, is similar to the TD-Signal method except that distance between door entrances is computed in frequency domain and not in time domain. To identify the person, first the time window of door acceleration containing the entire door opening event is extracted. 
Second, the fast Fourier transform of the window is computed. To compute the distance between the identified door entry and a labeled door entry, Manhattan distance, i.e. the sum of absolute differences in magnitude for each frequency, is used. The distance is to each labeled door entry is computed and the labels of the most similar entries are used to identify the person who entered the door. Similar to the TD-Signal method, the majority voting and weighted voting for 1 to 10 most similar entries (i.e. neighbors in the $k$-nn algorithm) was used. In addition, the maximal frequency was used to compute the Manhattan distance between FFT of two entries.

Figure 5 shows the Manhattan distances between the average FFTs for each person. It shows that there are five clusters of people with similar entries: people with IDs 4, 5 and 7 belong to the first cluster, and IDs 3, 2, and 11 to the second cluster, which is quite similar to the first. The person with ID 1 is the only one in the third cluster; IDs 6, 10, and 8 belong to the fourth cluster. Finally, people with IDs 9 and 0 , which are very different from the rest of the people, belong to the fifth cluster. Observing this relations was the first indication that the FT-Signal method can be used to identify people when entering through the door. The full evaluation of the method is given in Section 5.2.

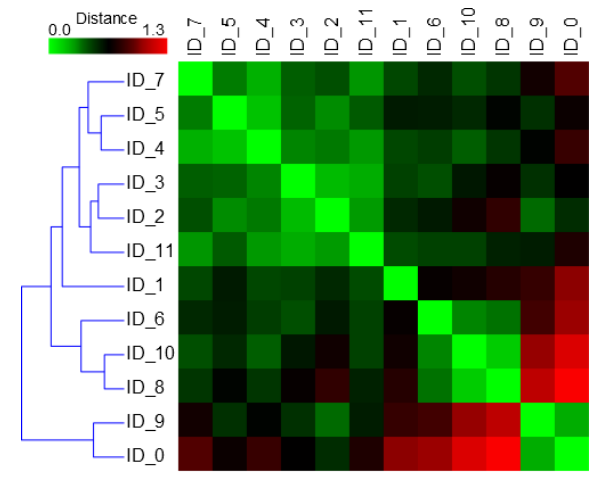

Figure 5: Distance between average entrance of each person according to the distance between the average FFT transforms of acceleration signals and hierarchical clustering of persons.

\section{$5 \quad$ Experiments}

The experiments were performed on a dataset recorded in an office. The office door was equipped with a 3-axis accelerometer fixed near the door handle. Total of 12 people (aged 21 to 87) were asked to enter and leave the office multiple times. A total of 1007 entries were recorder. In order to obtain unbiased recordings, the volunteers did not know that the goal was to record the movement of the door. Instead, they were asked to perform the following task: remember a password with a dozen of characters written on a paper in the office and then to re-write the password on a paper outside the office. During the recordings, an experiment supervisor labeled each entry using a smartphone, which was synchronized with the acceleration data. The supervisor marked the start and the end of each recording, and labelled each entry with the person's name. 
The evaluation of the methods was performed using leave-one-out cross-validation technique. That means each example was evaluated using the rest of the examples as a training set. This way, in each case the training data contained examples of the tested person, which is acceptable for our application because the system is supposed to recognize people which are already present in the database of recordings.

In the next two subsections the results achieved for the time domain and the frequency domain are presented. In the last Subsection 5.3, the methods for both domains are combined and the results for all of the combinations are presented.

\section{$5.1 \quad$ Time Domain}

Figure 6 shows the results for the time domain feature based method - TD-Feature. The accuracy achieved for each of the four classification algorithms is presented: Decision Tree j48, Random Forest, $k$-NN, and Support Vector Machine (SVM). The results show that the SVM is the best performing achieving $84.6 \%$ accuracy. We additionally checked the difference in accuracy using paired T-test with $p$-value of 0.05 . The statistical tests showed that $\mathrm{j} 48$ achieves significantly lower accuracy compared to the other three methods, and that the difference between the SVM, k-NN and Random Forest is not significant.

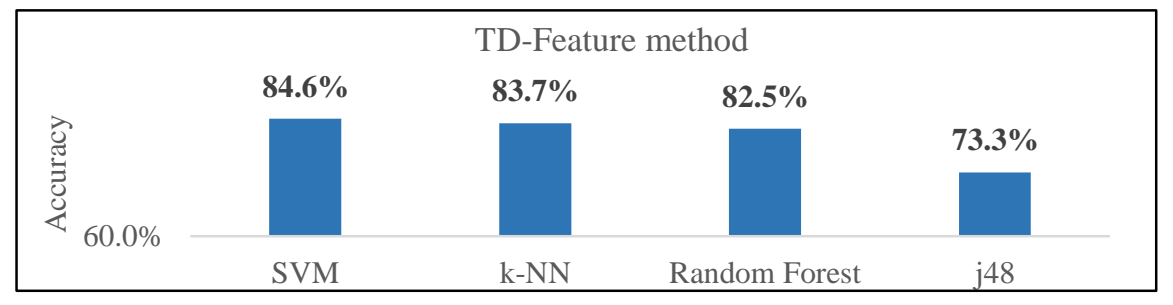

Figure 6. The classification performance (accuracy) achieved for the TD-Feature based method using four classification algorithms: Decision Tree j48, Random Forest, $k$-NN, and Support Vector Machine.

Figure 7 shows the identification accuracy of the TD-Signal method for various number of nearest neighbors. The identification accuracy of weighted voting approach was computed using 1 to 10 most similar entries. Using the single most similar entry results in $84.8 \%$ identification accuracy. For higher $k$ the identification accuracy generally increases until it reaches its maximum of $86.3 \%$ at $k=8$ and then starts decreasing. The differences in accuracy between different numbers of $k$ are not significant.

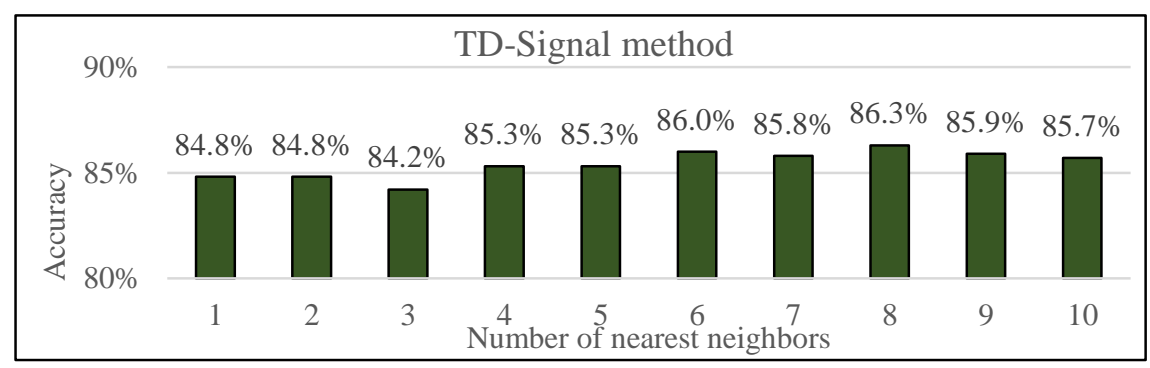

Figure 7. The accuracy achieved with the TD-Signal method using different number of nearest neighbors. 


\subsection{Frequency Domain}

Figure 8 shows the results for the FD-Feature method. The accuracy achieved for each of the four classification algorithms is presented: Decision Tree j48, Random Forest, $k$-NN, and SVM. The results show that the SVM performs the best, achieving $70.5 \%$ accuracy. Similar to the TF-Feature method, the statistical tests showed that j48 achieves significantly lower accuracy compared to the other three algorithms, and that the difference between the SVM, k-NN and Random Forest is not significant.

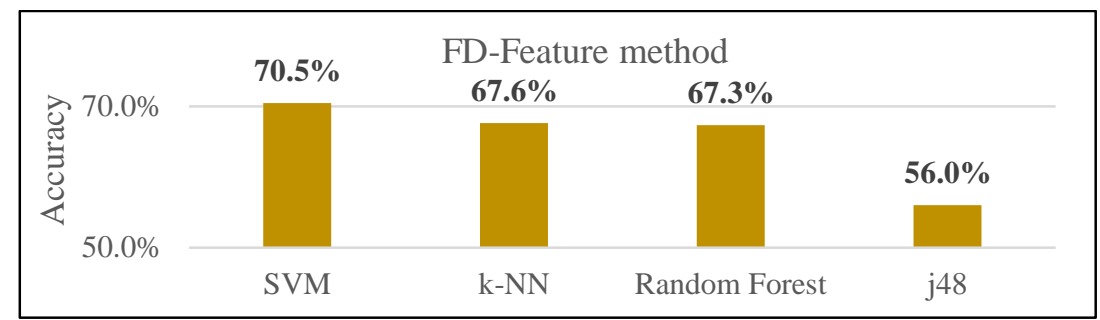

Figure 8. The classification performance (accuracy) achieved for the FD-Feature based method using four

classification algorithms: Decision Tree j48, Random Forest, $k$-NN, and Support Vector Machine.

Figure 9 shows the accuracy of the FD-Signal method for various number of nearest neighbors $(k)$ and different maximal frequencies. The accuracy for the weighted and majority voting approaches was computed using 1 to 10 most similar entries. Weighted voting performed better in each case compared to the majority voting. If $k$ is too low, the method is sensitive to noise and outliers. If $k$ is too high, the method blurs the boundary between classes (identities). The number of nearest neighbors where the maximum identification accuracy is reached depends on the maximal frequency used for comparison. The accuracy in general also depends on the maximal frequency. Similar as with the number of nearest neighbors, the accuracy increases with increasing frequency cut-off until it reaches the maximum at $50 \mathrm{~Hz}$ and then starts decreasing. If the maximal frequency used for comparison is too low, the method loses too much information and performs poorly. If it is too high, it gives more emphasis to high frequencies that do not enable distinguishing between identities of the entering persons.

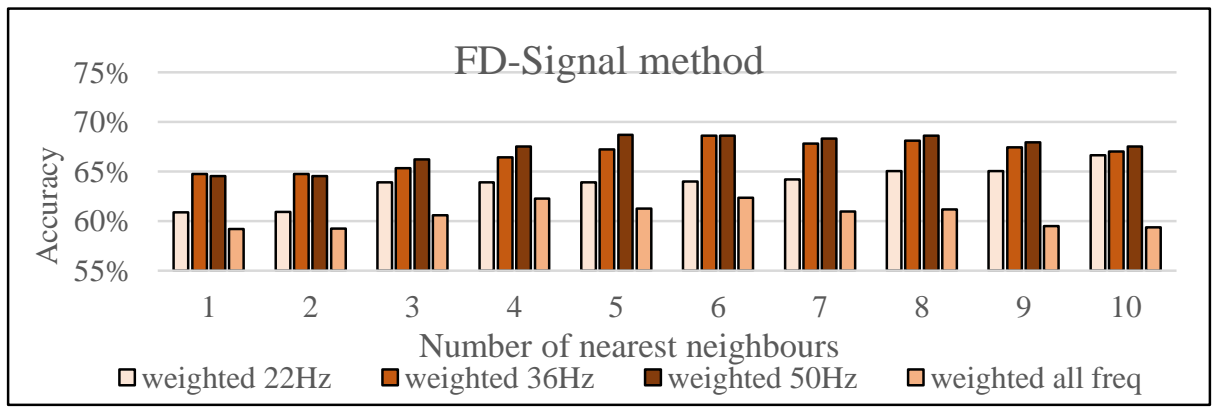

Figure 9. The accuracy achieved with the FD-Signal method using different number of nearest neighbors and different frequencies. 
The highest identification accuracy of $68.72 \%$ was achieved with 5 nearest neighbors, weighted voting and frequency cut-off at $50 \mathrm{~Hz}$.

\subsection{Combining the Time and Frequency Domain Methods}

Figure 10 summarizes the accuracy achieved by the best performing algorithm for each of the four methods: TD-Feature, TD-Signal, FD-Feature, and FD-Signal. It shows that the TD-Signal method performs the best, achieving $86.3 \%$. The statistical tests showed that this difference is not significant when the TD-Signal is compared to the TD-Feature and that it is significant compared to the other two. These results show that the representation of the acceleration data in time domain (as originally provided by the accelerometer) provides richer information about the person's signature of opening and closing a door.

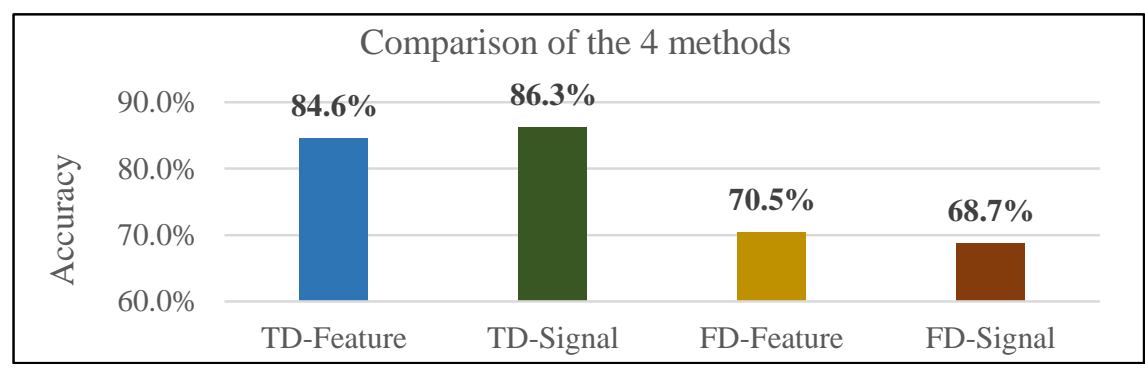

Figure 10. Comparison of the accuracy achieved by the four methods:

TD-Feature, TD-Signal, FD-Feature, and FD-Signal.

In the next step, we combined the four methods and exploited the 15 combinations, which are shown in Figure 11. The combination was achieved by combining the prediction outputs of each method. For each test instance, each method provides an array of prediction probabilities for each class value (for each person). This probabilities are summed for each of the combination methods and the class value (person) that has the biggest sum is chosen as the final one.

The results show that when the TD-Feature and TF-Signal are combined, the accuracy increases to $89.5 \%$, which is significantly better compared to each of the methods used individually, i.e., $84.6 \%$ and $86.3 \%$ respectively. The highest accuracy of $89.9 \%$ is achieved when the three methods are combined: TD-Feature, TF-Signal and FD-Feature.

Figure 12 shows the confusion matrix for the best performing combination, the combination of the three methods: TD-Feature, TF-Signal and FD-Feature, which achieved $89.9 \%$ accuracy. It also lists the recall and precision values for each person. The confusion matrix shows that person 10 has the lowest recall $(43.1 \%)$ and is often incorrectly identified as person 11. Similarly person pairs 4-5 and 6-8 have similar entries and are mutually misidentified. By analyzing per-person results (recall) one can note that for half of the people, i.e., 6 , the recall is above $95 \%$, which is a promising result, given that the recognition is performed using only the door acceleration. 


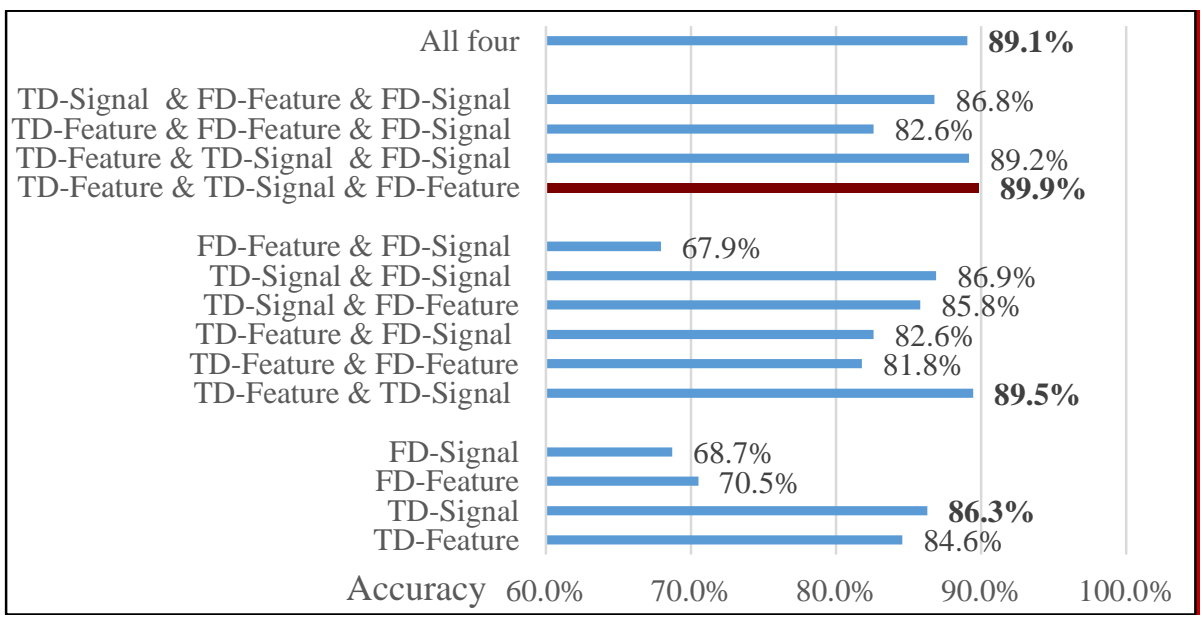

Figure 11. The accuracy achieved by combining the best performing algorithms for each of the 4 methods.

These results show that there is a considerable difference in identification accuracy for different people and that some people have similar ways of opening and closing the door, which in some cases makes the identification difficult.

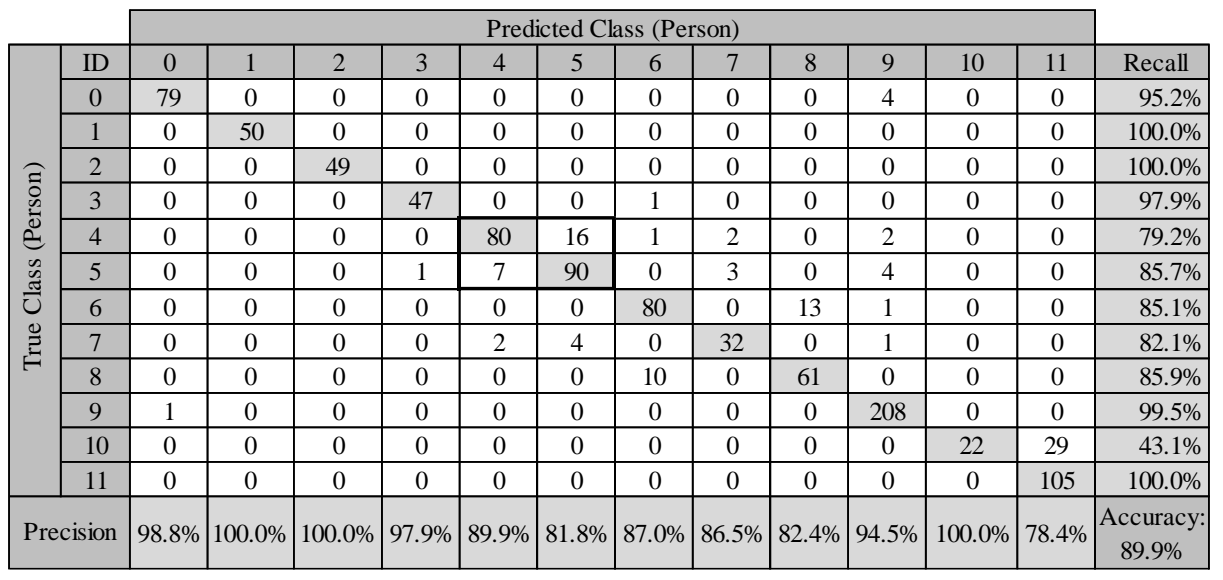

Figure 12. The confusion matrix for the best performing combination of three methods: TD-Feature, TFSignal and FD-Feature. Recall and precision are given for each person as well as overall accuracy.

\section{Conclusions}

The paper presented a novel unobtrusive approach to identify a person entering a room (e.g., home or office) using the acceleration produced by the door movement. To the best of our knowledge, our work is the first attempt to identify a person using door accelerations, and was also patented at the Slovenian national patent office [32]. Please note that the unobtrusiveness is regarding that the user does not have to wear 
any additional sensors or to perform any action to identify himself. However, our system still requires the user to pre-record certain number of entries.

The paper described four methods that recognize the person by analyzing the door acceleration data in time and frequency domain. For each domain we tested two types of methods: (i) feature-based - use features to describe the acceleration and then uses classification method to identify the person; (ii) signal-based - use the acceleration signal as input and find the most similar labeled entries in order to identify the person. The four methods were evaluated on a dataset of 1005 entrances recorded by 12 people. The results show that signal-based method that analyzes the signal in time domain performs best, achieving $86 \%$ accuracy. The comparison of the four methods (see Figure 10) shows that the methods that exploit the acceleration represented in time-domain perform significantly better compared to the methods that exploit the frequency domain. Therefore, one can conclude that the representation of the acceleration data in time domain (as originally provided by the accelerometer) provides richer information about the person's signature of opening and closing a door. Additionally, we combined the outputs of the four methods and examined all 15 combinations. Even by using simple technique for combination, i.e., weighted voting, the accuracy significantly increased up to $89.9 \%$. By analyzing per-person results (recall) one can note that for half of the people, i.e., 6 , the recall is above $95 \%$, which is a promising result, given that the recognition is performed using only the door acceleration. These results confirm that it is possible to identify a person with a system that is completely unobtrusive - no additional action such as scanning a fingerprint is required.

Some may argue that the identification accuracy is not high enough for a secure access-control applications, where almost perfect system is required. However, the proposed approach is novel and the results are promising for application when slightly lower accuracy is not problematic. Moreover, the approach is completely unobtrusive to the user and can therefore be used as an assistive technology or a smart-home sensor in offices and homes with limited number of people to enable smart-home automation personalization. After all, the goal of our system is not to substitute other reliable identification systems (e.g., fingerprint scanners), but to be used as an assistive technology (assist in daily tasks) and enable smart-home personalization in applications with a limited number of people (e.g., in a home or an office) by making the identification more comfortable, i.e. not requiring users to perform additional action. Therefore, a reasonable decrease in identification accuracy is tolerated in return for the increase in comfort of identification procedure.

One of the limitations of this study is that the data was recorded in a controlled lab scenario. Real-life poses challenges that are not addressed in this study, e.g., people sometimes enter in pairs or enter in a specific way, e.g., when carrying a heavy object. These issues need further attention before practical applications are considered.

For future work, we first plan to improve the identification accuracy by using more advanced combining approaches, such as meta-learning, Stacking [33], and Bayesian approach [34]. Another significant improvement should be achieved by classifying only members of a typical family or co-workers in an office. Our preliminary experiments show promising results [35]. We also plan to improve the computational performance of the DTW implementation by comparing the identified entrance signal 
only with the most representative labeled entries of each person instead of comparing with the entire database of examples. This can be done by clustering the entry signals of each person and using only the cluster centroids or by calculating an average entry signal to represent each person. Finally, we plan to test the methods on data recorded in real-life, e.g., by installing the system in an office and monitoring the office's entries and exits.

Acknowledgements. The authors would like to thank Tadej Vodopivec for recording the dataset and coding the initial version of software for data pre-processing and feature extraction. The authors would also like to thank mag. Borut Grošičar, for the discussions about the physics analysis of the door acceleration signal.

\section{References}

1. E. Aarts. Ambient intelligence: a multimedia perspective. IEEE Multimedia. 2004; 11(1):12-19.

2. DJ Cook, JC Augusto, VR Jakkula. Ambient intelligence: Technologies, applications, and opportunities. Pervasive and Mobile Computing. 2009; 5(4): 277-298.

3. K. Bashir, T. Xiang, S. Gong. Gait recognition without subject cooperation. Pattern Recognition Letters 31, 2052-2060, 2010.

4. L. Wang, T. Tan, H. Ning, W. Hu. Silhouette Analysis-Based Gait Recognition for Human Identification. IEEE Transactions on Pattern Analysis and Machine Intelligence, vol. 25, no. $12,2003$.

5. H. Gjoreski, M. Gams, M. Luštrek. Context-based fall detection and activity recognition using inertial and location sensors. Journal of Ambient Intelligence and Smart Environments, vol. 6, no. 4, p.p. 419-433 (2014).

6. Q. Li, J. Stankovic, M. Hanson, A.T. Barth, J. Lach and G. Zhou, Accurate, fast fall detection using gyroscopes and accelerometer-derived posture information. Proc. Sixth International Workshop on Wearable and Implantable Body Sensor Networks, 2009, pp. 138-143.

7. H. Gjoreski, S. Kozina, M. Gams, M. Luštrek, Álvarez-García JA, Hong JH, Ramos J, Dey AK, Bocca M, Patwari N. Competitive Live Evaluation of Activity-recognition Systems. IEEE Pervasive Computing, Vol:14, Issue: 1, pp. 70 - 77 (2015).

8. H. Gjoreski, S. Kozina, M. Luštrek and M. Gams. Using multiple contexts to distinguish standing from sitting with a single accelerometer. European Conference on Artificial Intelligence (ECAI), 2014.

9. N. Vyas, J. Farringdon, D. Andre, J. Stivoric. Machine learning and sensor fusion for estimating continuous energy expenditure. Proc. 23rd Conference on Innovative Applications of Artificial Intelligence, IAAI, 2011, San Francisco, CA, USA (2011), pp. 1613-1620.

10. H. Gjoreski, B. Kaluža, M. Gams, Radoje Milić, M. Luštrek. Context-based Ensemble Method for Human Energy Expenditure Estimation. Applied Soft Computing, in press (2015).

11. Patent application, Door monitoring system, patent no. WO 2011011282 A2.

12. L. Hong, Y. Wan, A. Jain. Fingerprint image enhancement: algorithm and performance evaluation. Journal Pattern Analysis and Machine Intelligence, IEEE Transactions on, 1998, Volume 20 Issue 8 Pages 777-789

13. Apple pay: https://www.apple.com/apple-pay/ 
14. M.A. Turk, A.P. Pentland. Face recognition using eigenfaces, Computer Vision and Pattern Recognition, 1991. Proceedings CVPR '91., IEEE Computer Society Conference on , vol., no., pp.586,591, 3-6 Jun 1991.

15. A. Juels. RFID security and privacy: a research survey, Selected Areas in Communications, IEEE Journal on, vol.24, no.2, pp.381,394, Feb. 2006

16. R. Piltaver, H. Gjoreski, M. Gams, Person Identification using Door Accelerations. AITAmI'15 Workshop, IJCAI, 2015, accepted.

17. R. Kohavi, H. George. Wrappers for feature subset selection. Artificial Intelligence. 97(12):273-324, 1997.

18. R. Quinlan. C4.5: Programs for Machine Learning. Morgan Kaufmann Publishers, San Mateo, CA, 1993.

19. L Breiman. Random Forests. Machine Learning. 45(1):5-32, 2001

20. D. Aha, D. Kibler (1991). Instance-based learning algorithms. Machine Learning. 6:37-66.

21. J. Platt: Fast Training of Support Vector Machines using Sequential Minimal Optimization. In B. Schoelkopf and C. Burges and A. Smola, editors, Advances in Kernel Methods - Support Vector Learning, 1998.

22. R. Bellman and R. Kalaba. On adaptive control processes. Automatic Control, IRE Transactions on, vol. 4, no. 2, pp. 1-9, 1959.

23. M. Hall, E. Frank, G. Holmes, B. Pfahringer, P. Reutemann, I.H. Witten. The WEKA Data Mining Software: An Update. SIGKDD Explorations, 2009, vol. 11 issue 1, pages 1018.

24. H. Sakoe, S. Chiba "Dynamic programming algorithm optimization for spoken word recognition," Acoustics, Speech and Signal Processing, 19 IEEE Transactions on, vol. 26, no. 1 , pp. 43-49, 1978.

25. C. C. Tappert, C. Y. Suen, and T. Wakahara, "The state of the art in online handwriting recognition," Pattern Analysis and Machine Intelligence, IEEE Transactions on, vol. 12, no. 8, pp. 787-808, 1990.

26. A. Kuzmanic and V. Zanchi, "Hand shape classification using dtw and lcss as similarity measures for vision-based gesture recognition system," in EUROCON, 2007. The International Conference on "Computer as a Tool", 2007, pp. 264-269.

27. T. Warren Liao, Clustering of time series data-a survey, Pattern Recognition, Volume 38, Issue 11, November 2005, Pages 1857-1874.

28. S. Salvador, P. Chan, FastDTW: Toward Accurate Dynamic Time Warping in Linear Time and Space. KDD Workshop on Mining Temporal and Sequential Data, pp. 70-80, 2004

29. G. Al-Naymat, S. Chawla, J. Taheri. SparseDTW: A Novel Approach to Speed up Dynamic Time Warping, 2012.

30. James W. Cooley and John W. Tukey. An algorithm for the machine calculation of complex Fourier series. Mathematics of Computaiton 19 (1965), pp. 297-301

31. A. Jakulin, "Machine Learning Based on Attribute Interactions." PhD Dissertation, 2005.

32. M. Gams, R. Piltaver, H. Gjoreski. Postopek identifikacije osebe, ki vstopa v prostor, patent P-201300281, Slovenian Intellectual Property Office, filed: September 19, 2013.

33. D. Wolpert. Stacked Generalization., Neural Networks, 5(2), pp. 241-259, 1992.

34. A.L. C. Bailer-Jones, K. Smith. Combining probabilities. Gaia (GAIA-C8-TN-MPIA-CBJ053), Data Processing and Analysis Consortium (DPAC), issue 2, 2011.

35. R. Piltaver, T. Vodopivec, M. Gams. Identifikacija oseb ob vstopu skozi vrata z uporabo pospeškomera in strojnega učenja. Proceedings of 16th International multiconference Information Society, vol A, pp. 90-93, 2013. 